von deutlich über 100.000 Euro erzielt. Vor diesem Hintergrund lässt der vom Kläger bezahlte Preis von 26.000 Euro keine Interpretation dahin zu, der Beklagte habe dafür einstehen wollen, das Bild sei unter allen Umständen im Kunsthandel jederzeit als Werk von Boudin erfolgreich zu veräußern, und er garantiere dies. Es gibt auch keine Garantie des Beklagten dafür, dass der Kläger das Bild kurzfristig mit einem Gewinn von 80.000 Euro über ein Auktionshaus versteigern kann oder dass eine Anerkennung durch den Herausgeber des Werkverzeichnisses von Boudin, so es eines gebe, gesichert ist. Im Rahmen der Vertragsverhandlungen war stets nur die Expertise von Prof. Dr. Z. angesprochen.

Schließlich ist ergänzend festzuhalten, dass es auch keine Anhaltspunkte dafür gibt, der Beklagte hätte für ein lediglich durch die Expertise von Prof. Z. Boudin zugeschriebenes Werk einen sittenwidrig überhöhten Kaufpreis verlangt, zumal auch der gerichtlich beauftragte Sachverständige Prof. Dr. L. seine Überzeugung davon, dass das Gemälde aus der Hand von Boudin stammt, nachvollziehbar und plausibel dargelegt hat und die maltechnisch-naturwissenschaftliche Untersuchung von Privatdozentin Dr. H. S. dies stützt, dem Gutachten von Prof. Dr. C. L. jedenfalls nicht entgegensteht.

\section{Berufung des Beklagten}

Ansprüche des Klägers gegen den Beklagten im Zusammenhang mit dem Erwerb des Gemäldes bestehen daher insgesamt nicht. Bei dieser Sachlage war - neben der Zurückweisung der Berufung des Klägers - auf die Berufung des Beklagten das Urteil des Landgerichts, soweit es der Klage stattgegeben hat, zu ändern und die Klage insgesamt abzuweisen. (Entscheidung von der Redaktion bearbeitet.)

\title{
Der Museumsbegriff im Umsatzsteuerrecht
}

Oberverwaltungsgericht Nordrhein-Westfalen, Urteil vom 31. Juli 2013 - 14 A 457/13

Eine Sammlung ist für ein Museum ein Einrichtungs- und kein Aufgabenmerkmal. Zur Bestimmung des Museumsbegriffs nach \$ 4 Nr. 20 a USt G kann es daher dahinstehen, ob eine eigene oder ständige Sammlung vorliegt, solange gleiche kulturelle Aufgaben wie von Museen in öffentlich-rechtlicher Trägerschaft erfüllt werden. (Leitsatz der Redaktion)

\section{Tatbestand}

- Die Klägerin betreibt die am 19. Juni 1992 eröffnete Kunstund Ausstellungshalle der Bundesrepublik Deutschland - KAH - in Bonn.

In $\S 2$ Abs. 1 des Gesellschaftsvertrages wird der Gegenstand der Gesellschaft u.a. wie folgt definiert:

Gegenstand des Unternehmens ist es, die Kunst- und Ausstellungshalle der Bundesrepublik Deutschland zu betreiben und geistige und kulturelle Entwicklungen von nationaler und internationaler Bedeutung sichtbar zu machen, insbesondere durch Ausstellungen, die von der Gesellschaft veranstaltet oder - auch im Austausch - übernommen werden.

Auf Antrag der Klägerin bescheinigte die Bezirksregierung Köln mit Bescheid vom 6. März 1995, dass die KAH die gleichen kulturellen Aufgaben erfüllt wie die in $\S 4 \mathrm{Nr}$. 20 Buchst. a) S. 2 des Umsatzsteuergesetzes - UStG - genannten staatlichen und kommunalen Einrichtungen. Die Bescheinigung enthielt den Zusatz: „Sie wird auf Widerruf erteilt".

Mit Schreiben vom 4. März 2009 stellte die Klägerin den Antrag, die Bescheinigung zu widerrufen. Zur Begründung führte sie im Wesentlichen aus: Die Klägerin sei kein Museum, denn sie verfüge weder über eine wissenschaftliche Sammlung noch über eine Kunstsammlung. Die Ausstellungen würden gemeinsam mit Künstlern, Galeristen und privaten Leihgebern konzipiert und durchgeführt, wobei Leihgaben von in- und ausländischen Leihgebern für die Dauer der Ausstellung ausgeliehen würden. Darüber hinaus konzipiere und projektiere die Klägerin Tourneeveranstaltungen für verschiedene Ausstellungen, d.h. die von der Klägerin erarbeiteten Ausstellungskonzepte würden auch in anderen vergleichbaren Ausstellungshäusern gezeigt. Sie erfülle daher keineswegs die gleichen kulturellen Aufgaben wie die in $\S 4 \mathrm{Nr}$. 20 Buchst. a) UStG genannten staatlichen oder kommunalen Einrichtungen. Die Gleichstellung der Klägerin mit diesen Einrichtungen führe zu einem erheblichen 
Wettbewerbsnachteil gegenüber gleichartigen Einrichtungen, da die von der Klägerin gezahlte Vorsteuer für die von ihr eingekauften Waren und Dienstleistungen nicht geltend gemacht werden könne.

Mit Bescheid vom 27. Mai 2009 lehnte die Bezirksregierung Köln den Antrag der Klägerin auf Widerruf der Bescheinigung ab und führte aus, ein Widerruf der Bescheinigung vom 6. März 1995 komme nicht Betracht, da die Voraussetzungen für deren Erteilung unverändert vorlägen. In der KAH würden Kunstgegenstände und Kunstsammlungen ausgestellt und dadurch der Öffentlichkeit zum Betrachten und zu den damit verbundenen kulturellen Zwecken zugänglich gemacht, sodass die Klägerin damit die gleichen kulturellen Aufgaben wie ein Museum in öffentlich-rechtlicher Trägerschaft erfülle.

Die Klägerin hat am 25. Juni 2009 Klage erhoben und zur Begründung im Wesentlichen vorgetragen: Das beklagte Land sei verpflichtet, die Bescheinigung vom 6. März 1995 zu widerrufen, da die KAH weder nach dem Gesellschaftsvertrag noch nach dem tatsächlichen Handeln ein Museum sei. Nach der Gründung der KAH habe der Tätigkeitsschwerpunkt in der zeitlich begrenzten Darbietung von Schausammlungen international anerkannter Museen bestanden. Zwischenzeitlich habe sich die KAH jedoch zu einem Dienstleistungsunternehmen weiterentwickelt, das unter Nutzung des eigenen wissenschaftlichen Apparates Ausstellungen mit Leihgaben unterschiedlichster Leihgeber - insbesondere privater Sammler, Galerien, ausländischer Institutionen, Kirchen und weiterer nicht musealer Einrichtungen - konzipiere und gestalte. Die kunsthistorischen Ausstellungen würden an Einrichtungen im In- und Ausland weitergegeben, wobei die KAH bei ihrer Tätigkeit auf die Zuarbeit einer wachsenden Zahl von Fremdfirmen angewiesen sei.

Zudem müsse der Museumsbegriff nicht aus dem allgemeinen Sprachgebrauch abgeleitet werden, da $\S 4$ Nr. 20 Buchst. a) S. 4 (Anm.: in der bis zum 31. Dezember 2010 geltenden Fassung S. 3) UStG eine spezifische Legaldefinition - wissenschaftliche Sammlungen und Kunstsammlungen - für ein Museum enthalte. Aufgabe des Museums sei das Sammeln, Bewahren, Erforschen, Ausstellen und Vermitteln von kunst- bzw. kulturwissenschaftlichen Exponaten; das Sammeln gehe dabei dem Ausstellen voraus. Allein die Präsentation des zuvor Gesammelten in einer Ausstellung erfülle den Museumszweck. Dies habe zur Folge, dass nur das Vorhandensein einer eigenen Sammlung eine Museumseigenschaft begründen könne. Museum könne nur die Sammlung selbst sein, nicht aber der vorübergehende Ausstellungsraum. Als Sammlung sei nur die dauerhafte Zusammenführung von Objekten zu einer thematischen Einheit anzusehen. Die lediglich temporäre Darbietung fremder Exponate im Rahmen einer Ausstellung könne nicht selbst Sammlung sein, da die gezeigten Objekte Bestandteile der Herkunftssammlung bleiben und nach dem Ende der Ausstellung dem Einflussbereich des Ausstellers wieder gänzlich entzogen würden. Bei der Prüfung der Gleichartigkeit der kulturellen Aufgabenerfüllung durch das beklagte Land sei zudem zu beachten, dass diese nicht losgelöst vom Museumsbegriff und der Legaldefinition des $\S 4 \mathrm{Nr}$. 20 Buchst. a) S. 4 UStG erfolgen könne. Es könnten und dürften nur solche Einrichtungen als gleichartig bewertet werden, die Sammlungen seien, in denen wertvoller Kunst- oder Kulturbesitz, insbesondere kulturgeschichtlicher, kunstgeschichtlicher oder naturwissenschaftlicher Art, der Allgemeinheit erhalten und zugänglich gemacht werde. Rein vorsorglich werde vorgetragen, dass die $\mathrm{KAH}$ auch ihrer katalogisierten Bibliothek und des Archivs wegen keine wissenschaftliche Sammlung sei. Zweck sei es gerade nicht, kunst- oder kulturgeschichtliche Gegenstände mit dem Ziel zu sammeln, sie der Allgemeinheit zu erhalten. Es handle sich bei diesen beiden „Einrichtungen“ lediglich um unselbständige Annexe der im Gesellschaftsvertrag allein festgelegten Aufgabe, Ausstellungen zu veranstalten und zu übernehmen.

\section{Die Klägerin hat beantragt}

das beklagte Land unter Aufhebung seines ablehnenden Bescheides vom 27. Mai 2009 zu verpflichten, die Bescheinigung der Beklagten nach § 4 Nr. 20 Buchst. a) UStG vom 16. März 1995 ab März 2009 zu widerrufen.

\section{Das beklagte Land hat beantragt,}

die Klage abzuweisen.

Es hat zur Begründung im Wesentlichen vorgetragen, es prüfe im Rahmen des $\S 4 \mathrm{Nr}$. 20 Buchst. a) UStG innerhalb ihrer Zuständigkeit nicht die Gleichartigkeit der KAH mit einem Museum, sondern bescheinige ausschließlich, ob die KAH die gleichen kulturellen Aufgaben wie ein Museum erfülle. Ersteres werde allein von der Finanzbehörde im Rahmen ihrer Zuständigkeit geprüft. Der Klägerin sei allerdings darin beizupflichten, dass die Frage der Vergleichbarkeit der kulturellen Aufgaben nicht völlig losgelöst von dem in $\S 4 \mathrm{Nr}$. 20 Buchst. a) S. 4 UStG normierten Museumsbegriff zu beantworten sei.

Unter Bezugnahme auf eine von ihm eingeholte Stellungnahme des Kunsthistorikers Dr. M. vom 18. Februar 2010 hat das beklagte Land aber darauf verwiesen, dass ein Museum nicht zwingend über eine eigene Sammlung definiert sei, sondern stattdessen auch die Ausstellung von Kunst ausreiche, um als Kunstsammlung eingestuft zu werden. Indem die $\mathrm{KAH}$ ausschließlich Wechselausstellungen mit Hilfe externer Leihgeber konzipiere und präsentiere, erfülle sie die gleichen kulturellen Aufgaben wie ein Museum in öffentlich-rechtlicher Trägerschaft. Es handele sich bei der KAH um eine Spielart des klassischen Museums, die sich auf die drei wesentlichen Teile - Präsentation, Vermittlung und Erforschung - in Form von publikumsträchtigen „Blockbuster-Ausstellungen“ konzentriere. Selbst bei Museen mit eigener Sammlung sei eine Entwicklung hin zu einer vermehrten Wechselausstellungstätigkeit festzustellen. 
Weiterhin halte die Klägerin mit ihrer Bibliothek und ihrem Archiv eine wissenschaftliche Sammlung im Sinne des § 4 Nr. 20 Buchst. a) S. 4 UStG vor. Der Klägerin sei zwar darin beizupflichten, dass das Vorhandensein von Bibliothek und Archiv allein nicht ausreichend sei, um eine abschließende Aussage zur Vergleichbarkeit der kulturellen Aufgabenwahrnehmung unter dem Aspekt des "Museums" zu treffen. Es sei aber auch nicht zutreffend, diese beiden Einrichtungen bei der Betrachtung außen vor zu lassen.

Schließlich ergebe sich auch aus dem vorgelegten Gesellschaftsvertrag, insbesondere aus § 2 Abs. 1, der im Laufe der Zeit unverändert geblieben sei, gerade keine Änderung der Aufgabenwahrnehmung der Klägerin gegenüber dem Zeitpunkt, zu dem die streitige Bescheinigung erteilt worden sei.

Durch das angefochtene Urteil, auf das Bezug genommen wird, hat das Verwaltungsgericht die Klage abgewiesen, allerdings die Berufung zugelassen.

Die rechtzeitig eingelegte Berufung begründet die Klägerin wie folgt: Das Verwaltungsgericht stufe die Bescheinigung vom 6. März 1995 zutreffend als Verwaltungsakt mit Doppelcharakter ein, der einerseits begünstigend wirke, weil er eine Befreiung von der Umsatzsteuerpflicht nach sich gezogen habe, und andererseits - als Kehrseite dieser Steuerbefreiung - einen Vorsteuerabzug ausschließe. Die Bescheinigung sei zudem ein Verwaltungsakt mit Dauerwirkung, weil die Rechtsfolge der Befreiung von der Umsatzsteuer so lange bestehen bleibe, wie der Bescheid Gültigkeit behalte. Ihr, der Klägerin, stehe jedoch ein Anspruch auf Widerruf der Bescheinigung zu, weil sie sich jedenfalls zum Zeitpunkt der beantragten Aufhebung sowohl aus formellen als auch aus materiellen Gründen als rechtsfehlerhaft erweise. Zunächst führe ihr Antrag auf Widerruf der Bescheinigung zum Wegfall der formellen Voraussetzungen. Denn die Bescheinigung nach $\S 4$ Nr. 20 Buchst. a) S. 2 UStG könne nur auf Antrag erteilt werden. Antragsberechtigt seien sowohl die Einrichtung als auch die zuständige Finanzbehörde. Für die Erteilung der Bescheinigung könne auf einen Antrag nicht verzichtet werden. Mit dem Antrag auf Widerruf, der als actus contrarius zur ursprünglichen Beantragung der Bescheinigung im Jahr 1995 zu werten sei, sei der ausgestellten Bescheinigung für die Zukunft ihre formelle Legitimation entzogen. Die Bescheinigung verstoße auch gegen materielles Recht, da die gesetzlichen Voraussetzungen für ihre Erteilung durch die Klägerin nicht (mehr) erfüllt würden. Das Verwaltungsgericht gehe fehl, wenn es die Auffassung vertrete, dass die Frage der Gleichartigkeit mit einem Museum von den Finanzbehörden und gegebenenfalls von den Finanzgerichten zu prüfen sei. Die Frage, ob die KAH umsatzsteuerrechtlich die gleichen Aufgaben wie ein Museum in öffentlich-rechtlicher Trägerschaft erfülle, könne nicht losgelöst vom Museumsbegriff in § 4 Nr. 20 Buchst. a) S. 4 UStG gesehen werden. Entscheidungsrelevant sei die Frage, ob die KAH die gleichen kulturellen Aufgaben wie ein Museum erfülle. Maßstab für die Prüfung sei ausschließlich der Museumsbegriff des $\S 4$ Nr. 20 Buchst. a) S. 4 UStG, wonach
Museen im Sinne dieser Vorschrift wissenschaftliche Sammlungen und Kunstsammlungen seien. Insoweit setze zunächst das Verwaltungsgericht zu Unrecht eine Kunstausstellung mit einer Kunstsammlung gleich. Zudem könne der Museumsbegriff nur dann erfüllt werden, wenn es sich um eine eigene Sammlung handele. Ansonsten würden auch Kunstgalerien oder Kunstmessen dem Museumsbegriff im Sinne der gesetzlichen Definition unterfallen. Sinn und Zweck der mit der Steuerbefreiung verbundenen Gleichbehandlung von Einrichtungen der öffentlichen Hand mit gleichartigen Einrichtungen anderer Unternehmer würden es gebieten, die steuerliche Entlastung auf Museen mit eigenen Sammlungen zu beschränken. Mit der KAH vergleichbare Einrichtungen in anderen Bundesländern seien von der Umsatzsteuer nicht befreit. Wenn das beklagte Land, wie in seiner Stellungnahme vom 18. Februar 2010 ausgeführt, die Auffassung vertrete, die KAH erfülle bis auf das Sammeln und Bewahren die gleichen kulturellen Aufgaben bzw. die wesentlichen kulturellen Aufgaben eines Museums, so weiche sie von der Museumsdefinition des $\S 4$ Nr. 20 Buchst. a) S. 4 UStG ab. Dass auch öffentliche Museen in verstärktem Maße dazu übergingen, in ihren Häusern wechselnde Ausstellungen in Form von Sonderausstellungen zu zeigen, die entweder komplett aus den Sammlungsbeständen anderer Einrichtungen oder privater Leihgeber zusammengestellt seien oder aber nur zu einem geringen Anteil aus der eigenen Sammlung bestückt würden, habe keinen Begründungswert für die Argumentation im vorliegenden Klageverfahren. Dem Verwaltungsgericht sei auch insoweit nicht zu folgen, als es die Auffassung vertrete, der Anspruch der Klägerin auf Widerruf der Bescheinigung ergebe sich auch deshalb nicht, weil keine Änderung der Sachund Rechtslage eingetreten sei und die Bescheinigung auch heute noch in gleicher Weise erteilt werden müsse. Es sei eine Veränderung der Sachlage eingetreten, da sich der Aufgabenschwerpunkt der Klägerin verlagert habe und hierdurch eine andere Kostenstruktur entstanden sei. Angesichts der veränderten Umstände bestehe auch entgegen der Auffassung des Verwaltungsgerichts ein Anspruch auf Wiederaufgreifen des Verfahrens gemäß $§ 51$ Abs. 1 Nr. 1 VwVfG NRW. Es liege eine Veränderung der Sachlage vor, die im Nachhinein zugunsten der Klägerin zu berücksichtigen sei. In der mündlichen Verhandlung vor dem Senat hat die Klägerin ihren Vortrag dahin ergänzt, dass eine Bescheinigung nach § 4 Nr. 20 Buchst. a) S. 2 UStG auch deshalb rechtmäßig nicht erteilt werden könne, weil die Klägerin kein anderer Unternehmer als die in S. 1 der Vorschriften genannten sei. Es handele sich bei der Klägerin um eine Einrichtung des Bundes. Das ergebe sich nicht nur aus den gesellschaftsrechtlichen Stimmverhältnissen, die zu 60\% beim Bund und nur zu $40 \%$ bei den Ländern lägen, sondern auch aus dem Umstand, dass die Klägerin vollständig vom Bund finanziert und auch in dessen Haushalt geführt werde. Daher komme für die Klägerin als Einrichtung des Bundes lediglich eine gesetzliche Umsatzsteuerbefreiung nach S. 1 der Vorschrift in Betracht, die aber nicht eingreife, da es am Tatbestandsmerkmal Museum fehle. Das müsse auch im Rahmen der Erteilung einer Bescheinigung nach S. 2 der Vorschrift beachtet werden, 
weil sonst verfassungswidrig kein Rechtschutz gegen eine aufgedrängte Bescheinigung gewährt werde, obwohl schon die Anwendbarkeitsvoraussetzungen der Vorschrift fehlten.

Die Klägerin beantragt,

das angegriffene Urteil zu ändern und nach dem erstinstanzlichen Klageantrag zu erkennen.

Das beklagte Land beantragt,

die Berufung zurückzuweisen.

Es hält die Ausführungen des Verwaltungsgerichts im erstinstanzlichen Urteil für zutreffend und weist ergänzend darauf hin, der Klägerin stehe kein Anspruch auf Aufhebung der Bescheinigung zu. Eine Änderung der Sachlage sei nicht eingetreten. Die Voraussetzungen für die Erteilung der Bescheinigung bestünden auch weiterhin. In dem Antrag auf Widerruf liege keine "Art" der auflösenden Bedingung durch Veränderung der Sachlage. Es bestehe auch kein Anspruch auf Wiederaufgreifen des Verfahrens. Angesichts der Ausführungen der Klägerin in der mündlichen Verhandlung vor dem Verwaltungsgericht am 19. September 2012, dass sich der Tätigkeitsbereich der KAH dem Grunde nach seit Eröffnung nicht geändert habe, und unter Berücksichtigung der unverändert geltenden Vorgaben des Gesellschaftsvertrages habe die Bescheinigung weiterhin Geltung. Soweit es die Frage der "Gleichartigkeit" betreffe, sei zu unterscheiden zwischen der Prüfung der Kultusverwaltung, ob das Unternehmen die "gleichen kulturellen Aufgaben [...] erfülle" wie die in $\S 4 \mathrm{Nr}$. 20 Buchst. a) S. 1 UStG bezeichneten Einrichtungen und der Prüfung, ob die Klägerin eine Einrichtung betreibe, die im Sinne des Umsatzsteuerrechts einem Museum in öffentlich-rechtlicher Trägerschaft gleichartig sei. Diese Prüfung sei ausdrücklich dem laufenden Besteuerungsverfahren vorbehalten. Eine umfassende Prüfung der Gleichartigkeit durch die Kultusbehörde würde auch zu einer unzulässigen Bindung der Finanzbehörden führen.

Wegen der weiteren Einzelheiten des Sachverhalts und des Parteivorbringen im Übrigen wird auf den Inhalt der Verfahrensakte und der beigezogenen Verwaltungsvorgänge Bezug genommen.

\section{Entscheidungsgründe}

Die Berufung ist zulässig.

Sie ist jedoch nicht begründet. Das Verwaltungsgericht hat die Klage zu Recht abgewiesen. Der Klägerin steht der geltend gemachte Anspruch auf Widerruf (Aufhebung) der Bescheinigung vom 6. März 1995 für einen Zeitraum beginnend ab März 2009 nicht zu.
Wie bereits das Verwaltungsgericht im erstinstanzlichen Urteil geht der Senat entsprechend dem Klagebegehren davon aus, dass die Klägerin ihren Anspruch abweichend vom Wortlaut des gestellten Klageantrages nicht auf einen Widerruf der Bescheinigung beschränkt, sondern unabhängig von der rechtlichen Konstruktion deren Aufhebung begehrt. Dies entspricht auch den Erörterungen in der mündlichen Verhandlung vom 31. Juli 2013 und wird im Übrigen vom beklagten Land nicht in Zweifel gezogen.

Das so umschriebene Klagebegehren hat keinen Erfolg.

Insoweit kann dahinstehen, ob der - wohl zutreffenden Auffassung des Verwaltungsgerichts zu folgen ist, maßgeblich seien die Regelungen der $\S 49$ (Widerruf), 48 (Rücknahme) und 51 (Wiederaufgreifen) des Verwaltungsverfahrensgesetzes für das Land Nordrhein-Westfalen - VwVfG NRW - und nicht die der §§ 131 (Widerruf) oder 130 (Rücknahme) der Abgabenordnung - AO -, gegebenenfalls unter Heranziehung des $\S 580$ ZPO (Wiederaufgreifen), in entsprechender Anwendung. Vgl. zum Letzteren: Rüsken, Klein, AO, 11. Aufl., § 130 Rn. 130.

Denn unabhängig von der Frage, welche der genannten Rechtsnormen überhaupt zur Erreichung des klägerischen Rechtsschutzzieles anwendbar ist und welcher rechtliche Weg konstruktiv einschlägig ist, würde ein Anspruch auf Aufhebung (Beseitigung) der Bescheinigung in jedem Fall voraussetzen, dass heute die Voraussetzungen für die Erteilung der Bescheinigung nicht - mehr - vorliegen. Dies lässt sich jedoch, wie es das Verwaltungsgericht im erstinstanzlichen Urteil zutreffend ausgeführt hat, nicht feststellen.

Entgegen der Auffassung der Klägerin ist der Bescheinigung nicht bereits dadurch der Boden entzogen worden, dass im Antrag auf Widerruf bereits konkludent eine Rücknahme des im Jahr 1995 gestellten Antrags auf Erteilung der Bescheinigung enthalten wäre. Zwar mag im Antrag auf Widerruf auch eine Rücknahme des Antrags auf Erteilung der Bescheinigung gesehen werden können, ohne dass es insoweit einer ausdrücklich erklärten Antragsrücknahme bedurft hätte. Dies hat jedoch nicht zur Folge, dass bereits damit die Bescheinigung rechtswidrig oder unwirksam geworden wäre und sich das vorliegende Klageverfahren dann erledigt hätte - vgl. § 43 Abs. 2 VwVfG NRW/§ 124 Abs. 2 AO.

Dahinstehen kann, ob die Rücknahme verfahrensrechtlicher Anträge, wie hier des ursprünglich gestellten Antrages auf Erteilung der Bescheinigung, nur bis zur Bekanntgabe der Entscheidung zulässig ist, weil mit dem Erlass des Verwaltungsakts das Verwaltungsverfahren beendet ist. So: Stelkens/Bonk/SachsSchmitz, VwVfG, 7. Aufl., § 22 Rn. 67 ff. (mwN), oder ob ein Antrag noch bis zum Eintritt der Unanfechtbarkeit der Entscheidung zurückgenommen werden kann, so: Kopp/Ramsauer, VwVfG, 13. Aufl., § 22, Rn. 85 (mwN). 
Jedenfalls hat die Bescheinigung vom 6. März 1995 Bestandskraft erlangt und ist damit unanfechtbar geworden.

Auch aus dem hier in Rede stehenden Fachrecht lässt sich nicht herleiten, dass ausnahmsweise eine Rücknahme des Antrags auf Erteilung der Bescheinigung selbst für die Zeit nach Eintritt deren Unanfechtbarkeit zulässig sein könnte.

Zwar ist der Klägerin zuzugestehen, dass das Verwaltungsverfahren auf Erteilung der Bescheinigung (zumindest in der Regel) auf Antrag des Unternehmers, der die Bescheinigung begehrt, oder der Finanzbehörde eingeleitet wird. Daraus folgt jedoch nicht, dass ein derartiger Antrag über die Rechtsfolge des Beginns des Verwaltungsverfahrens hinaus - vgl. § 22 S. 2 VwVfG NRW/§ 86 S. 2 AO - weitere Rechtsfolgen nach sich zieht. Bereits der Wortlaut der Norm des $§ 4$ Nr. 20 Buchst. a) S. 2 UStG bietet keine Anhaltspunkte dafür, dass der Antrag auf Erteilung der Bescheinigung ein Wirksamkeitserfordernis oder ein Erfordernis der Rechtmäßigkeit darstellen könnte. Vielmehr würde die Annahme eines Wirksamkeitserfordernisses geradezu der gesetzlichen Regelung des $\S 4$ Nr. 20 Buchst. a) S. 2 UStG widersprechen. Um den mit $\S 4$ Nr. 20 Buchst. a) S. 2 UStG geforderten "Nachweis" zu erbringen, bedarf es keiner Mitwirkung des Unternehmers, somit auch keines Antrags auf Erteilung der Bescheinigung. Dies folgt neben dem Wortlaut und der Entstehungsgeschichte der Norm auch aus der Systematik des Umsatzsteuerrechts. Mit der Einräumung eines Antragsrechts würde dem Unternehmer letztlich eine Möglichkeit eröffnet, auf die Steuerbefreiung zugunsten eines Vorsteuerabzuges zu verzichten, was mit der Regelung des $\S 9$ UStG unvereinbar wäre, weil $\S 4$ Nr. 20 Buchst. a) UStG nicht zu den in § 9 Abs. 1 UStG aufgezählten Steuerbefreiungstatbeständen gehört. Darüber hinaus zielt der Sinn der Norm darauf ab, die Wettbewerbsgleichheit zwischen den in $\S 4$ Nr. 20 Buchst. a) S. 1 UStG bezeichneten Einrichtungen der öffentlichen Hand mit den in S. 2 der Vorschrift beschriebenen gleichartigen Einrichtungen anderer Unternehmer herzustellen. Die Zielsetzung, Wettbewerbsneutralität der Umsatzbesteuerung sicherzustellen, würde konterkariert, wenn dem privaten Unternehmer insoweit ein Wahlrecht zugunsten eines im Einzelfall ihm günstigeren Vorsteuerabzugs eingeräumt würde, über das die unmittelbar kraft Gesetzes steuerbefreiten öffentlichen Unternehmer nach § 4 Nr. 20 Buchst. a) S. 1 UStG nicht verfügen. Eine Antragsbefugnis des privaten Unternehmers, die ihm die Option für eine Besteuerung einräumen würde, stünde schließlich auch nicht mit den gemeinschaftrechtlichen Vorgaben in Einklang. Vgl. zum Ganzen: BVerwG, Urteil v. 4. Mai 2006 - 10 C 10/05, u.a. DÖV 2006, 962 ff., [BVerwG 4.5.2006 - 10 C 10/05] [...], Rn. 15 ff.

Dem kann auch nicht mit dem von der Klägerin in der mündlichen Verhandlung vor dem Senat vorgebrachten Argument entgegengetreten werden, das Finanzamt habe es in der Hand, bei Wegfall des Antrags des Unternehmers selbst einen Antrag zu stellen. § 4 Nr. 20 Buchst. a) UStG erwähnt einen Antrag als notwendige Voraussetzung für eine rechtmäßige Bescheinigung der Kultusbehörde nicht. Nur rein tatsächlich wird die
Kultusbehörde nicht von Amts wegen tätig, weil sie - im Gegensatz zum Unternehmer und zum Finanzamt - am umsatzsteuerlichen Rechtsverhältnis nicht beteiligt ist. Jedenfalls die fehlende normative Verankerung eines Antragserfordernisses führt dazu, dass die Aufrechterhaltung eines früher gestellten Antrags keine Voraussetzung für die weitere Wirksamkeit oder Rechtmäßigkeit einer erteilten Bescheinigung ist.

Voraussetzung für eine Steuerbefreiung gemäß § 4 Nr. 20 Buchst. a) S. 2 UStG ist, dass es sich um Umsätze gleichartiger Einrichtungen anderer Unternehmer handelt, wenn die zuständige Landesbehörde bescheinigt, dass sie die gleichen kulturellen Aufgaben wie die in S. 1 bezeichneten Einrichtungen erfüllen. Dabei beschränkt sich die gerichtlich zu entscheidende Frage, ob die Bescheinigung rechtmäßig ist, ausschließlich auf den Aspekt der gleichen kulturellen Aufgaben. Die Regelung des $\S 4$ Nr. 20 Buchst. a) S. 2 UStG weist den Steuerbehörden einerseits und den Kultusbehörden andererseits verschiedene, voneinander abgrenzbare und sich nicht oder nur teilweise inhaltlich überschneidende Prüfungsaufgaben zu, nämlich einerseits die der Gleichartigkeit der Einrichtung und andererseits die der Gleichartigkeit der kulturellen Aufgaben. Sinn dieser Zweiteilung ist es, die Prüfung der Tatbestandsmerkmale für die Steuerbefreiung nicht allein den Finanzbehörden zu überlassen. Vielmehr hat der Gesetzgeber ein Bedürfnis gesehen, unter dem Gesichtspunkt einer größeren Sachkunde auch die Kultusbehörde einzuschalten. Soweit es allerdings für die Prüfung der Tatbestandsvoraussetzungen keiner spezifischen kulturellen Kenntnisse bedarf, verbleibt es bei der Entscheidung der für die Steuerbefreiung grundsätzlich zuständigen Finanzbehörde. Vgl. OVG Lüneburg, Urteil v. 3. Dezember 2008 - 2 LC 267/07, OVGE 52, 341, [...], Rn. 96 und 97.

Danach besteht kein Grund im Hinblick auf die für die Steuerbefreiung maßgeblichen Tatbestandsvoraussetzungen, dass bereits die Frage der Gleichartigkeit der Einrichtung durch die Kultusbehörde im Rahmen des Bescheinigungsverfahrens beantwortet wird. Denn für die Beantwortung dieser Frage bedarf es der besonderen Kompetenz der Kultusbehörde nicht. Es handelt sich vielmehr um spezifische steuerrechtliche Fragen, mit deren Klärung die Kultusbehörden außerhalb ihrer Kompetenzen nicht belastet werden sollen. Dies ist in der Rechtsprechung für das Tatbestandsmerkmal der Gleichartigkeit der Einrichtung, vgl. BVerwG, Beschluss v. 31. Juli 2008 - 9 B 80.07, NJW 2009, 793; [...], Rn. 4; Urteil v. 11. Oktober 2006 - 10 C 7.05, NJW 2007, 711,[...], Rn. 21 f., Urteil v. 11. Oktober 2006 - 10 C 4.06, NJW 2007, 714, [...], Rn. 13, 16; BFH, Urteil v. 4. Mai 2011 - XI R 44/08, BFHE 233, 367, [...], Rn. 15, und der Unternehmereigenschaft, vgl. BVerwG, Urteil v. 11. Oktober 2006 - 10 C 7.05, [...], Rn. 19, geklärt.

Nichts anderes hat zu gelten, soweit sich die Frage nach dem Tatbestandsmerkmal des anderen Unternehmers im Vergleich zu den in S. 1 genannten Unternehmern stellt. Auch für die Beantwortung dieser Frage bedarf es der besonderen Kompetenz 
der Kultusbehörden nicht, weil sie keinen spezifischen Bezug zu den kulturellen Aufgaben der in Rede stehenden Unternehmer aufweist.

Allenfalls wird man im Bescheinigungsverfahren etwa unter dem Gesichtspunkt fehlenden Rechtsschutzbedürfnisses zweifelsfreie Fälle ausscheiden können, etwa wenn es von vornherein offensichtlich ist, dass allein eine Einrichtung des Bundes, der Länder, der Gemeinden oder der Gemeindeverbände in Rede steht und daher eine Steuerbefreiung nur nach S. 1 in Betracht kommt. Dies lässt sich vorliegend für die Einrichtung der Klägerin nicht feststellen. Zwar handelt es sich bei der Klägerin um eine Gesellschaft mit beschränkter Haftung des Bundes und der Länder, sodass die Klägerin vollständig in der Hand von Bund und Ländern liegt. Es ist allerdings in Rechtsprechung und Literatur durchaus umstritten, ob gebietskörperschaftliche Eigengesellschaften der Regelung des $\S 4$ Nr. 20 Buchst. a) S. 1 UStG unterfallen. So hat der Bundesfinanzhof, vgl. Urteil v. 18. Mai 1988 - X R 11/82, BFHE 153, 459, [...], Rn. 18, entschieden, dass sich die Steuerfreiheit nach S. 1 auch auf Umsätze von solchen Theatern erstreckt, die in der Form einer privatrechtlichen Gesellschaft betrieben werden, vorausgesetzt, die Anteile an ihr gehören ausschließlich einer Körperschaft des öffentlichen Rechts und die Erträge fließen ausschließlich dieser Körperschaft zu. Die Kommentarliteratur zu dieser Frage ist gespalten. So wird die Auffassung vertreten, dass auch Gesellschaften mit beschränkter Haftung einer oder mehrerer Gebietskörperschaften nach S. 1 steuerbefreit sind. Vgl. Offerhaus/ Söhn/Lange-Kossack, UStG, Loseblattsammlung (Stand: Juni 2013), § 4 Nr. 20 Rn. 7; Rau/Dürrwächter-Schuhmann, UStG, Loseblattsammlung (Stand: März 2013), § 4 Nr. 20 Rn. 6; Hartmann/ Metzenmacher-Verweyen, UStG, Loseblattsammlung (Stand: Juni 2013), § 4 Rn. 10; Stadie, UStG, 2. Aufl., § 4 Nr. 20 Rn. 4.

Andererseits besteht die Auffassung, Eigengesellschaften von öffentlich-rechtlichen Körperschaften seien nicht nach S. 1 befreit, sondern könnten nur nach S. 2 befreit werden. Vgl. Vogel/Schwarz-Huschens, UStG, Loseblattsammlung (Stand: Juli 2013), § 4 Nr. 20 Rn. 26; Lippross, UStG, 23. Aufl., S. 672; Bunjes/ Geist-Heidner, UStG, 11. Aufl., § 4 Nr. 20 Rn. 3.

Daher ist dem Tatbestandsmerkmal des „anderen Unternehmers" hier im Bescheinigungsverfahren nicht weiter nachzugehen. Entgegen der Auffassung der Klägerin entsteht dadurch keine Rechtsschutzlücke. Der Klägerin ist es unbenommen, unter Bezugnahme auf die Auffassung, dass Eigengesellschaften von Gebietskörperschaften allein der gesetzlichen Steuerbefreiung nach $\S 4$ Nr. 20 Buchst. a) S. 1 UStG unterfallen, und die Meinung, sie betreibe kein Museum, Umsatzsteuer anzumelden und Vorsteuerabzug geltend zu machen und dazu, wenn das Finanzamt diese Rechtsauffassungen nicht teilt, um Rechtsschutz vor der Finanzgerichtsbarkeit nachzusuchen.

Entscheidend ist somit vorliegend allein die Frage, ob die Beklagte die gleichen kulturellen Aufgaben wie ein Museum in öffentlich-rechtlicher Trägerschaft erfüllt.
Die Frage nach der Gleichartigkeit der kulturellen Aufgaben ist - wie oben ausgeführt - von der Frage nach der Gleichartigkeit der Einrichtung zu trennen. Denn letztere entscheidet die Finanzbehörde in eigener Zuständigkeit, ohne dass insoweit unter dem Gesichtspunkt der größeren Sachkunde ein Bedürfnis zur Einschaltung der Kultusbehörden besteht. Zwar überschneiden sich beide "Gleichartigkeitsprüfungen" teilweise inhaltlich. Dennoch muss die Kultusverwaltung nicht mit einer abschließenden Beurteilung auch sämtlicher Fragen belastet werden, die sich im Rahmen der den Finanzbehörden zugewiesenen "Gleichartigkeitsprüfung" zusätzlich ergeben können. Vgl. BVerwG, Urteil v. 11. Oktober 2006 - 10 C 7.05, NJW 2007, 711, [...], Rn. 21/22; Urteil v. 11. Oktober 2006 - 10 C 4.06, NJW 2007, 714, [...], Rn. 16.

Zwar kann die Prüfung, ob die Klägerin mit ihrer Einrichtung die gleichen kulturellen Aufgaben erfüllt wie ein Museum in öffentlich-rechtlicher Trägerschaft, nicht losgelöst vom Museumsbegriff beurteilt werden. Vgl. BVerwG, Urteil v. 11. Oktober 2006 - 10 C 7.05, NJW 2007, 711, [...], Nr. 20.

Angesichts der genannten Aufgabenteilung zwischen Finanzbehörden einerseits und Kultusbehörden andererseits bedeutet dies aber nur, dass die Kultusbehörden den Museumsbegriff des § 4 Nr. 20 Buchst. a) S. 4 UStG in ihre Überlegungen einzustellen haben, soweit diesem Museumsbegriff Rückschlüsse auf die kulturellen Aufgaben zu entnehmen sind. Die Beurteilung, ob tatsächlich auch etwa nach seiner Organisationsstruktur ein Museum besteht, ist wiederum Aufgabe der Finanzverwaltung.

Dadurch stellt sich im vorliegenden, auf die Bescheinigung der Kultusbehörde beschränkten Verfahren zunächst die Frage, welche kulturellen Aufgaben ein Museum, das nach der Regelung des $\S 4$ Nr. 20 Buchst. a) S. 4 UStG als wissenschaftliche Sammlung oder Kunstsammlung definiert wird, erfüllt.

Bereits zu Beginn des 20. Jahrhunderts wurde von demselben Museumsbegriff ausgegangen, wonach Museen Sammlungen von Kunst-, Altertums- und Kulturgegenständen sind, um für die wissenschaftliche Forschung und für die Anschauung und Bildung des Publikums ein Gesamtbild der Kunst und Kultur der Vergangenheit und Gegenwart zu geben. Vgl. von Bitter, Handwörterbuch der Preußischen Verwaltung, 2. Bd., 1907, S. 123 (Stichwort: Museen).

Als Zweck von Sammlungen künstlerisch oder wissenschaftlich interessierender Gegenstände wurde ein doppelter angesehen. Einmal sollten sie der Kunst und Wissenschaft dienen, gleichzeitig aber sollten sie auch das Ziel einer allgemeinen Volksbildung fördern. Ersterem war nach der damaligen Auffassung nicht lediglich damit gedient, dass dem Künstler und Forscher ein möglichst umfangreiches Material in den Sammlungen zur Verfügung gestellt wurde. Vielmehr sollten die Museen hierüber hinaus eine insbesondere literarische Tätigkeit entfalten, indem sie für eine wissenschaftliche Bearbeitung 
der Sammlungsgegenstände Sorge trugen, indem sie ferner die notwendige Verbindung mit wissenschaftlichen Vereinen, Ausstellungen und Fachblättern, auch mit Privatsammlern suchten und pflegten und indem sie seitens ihrer höheren Beamten Reisen zu Studienzwecken und zur Teilnahme an Versteigerungen unternehmen ließen. Die Zwecke der Museen als Volksbildungsstätten sollten nicht ausschließlich dadurch erreicht werden, dass sie den Laien durch die Vorführung der toten Sammlungsgegenstände ein möglichst treues Bild der Entwicklung von Kunst und Kultur der Vergangenheit und Gegenwart zu geben suchten. In der Erkenntnis, dass das gesprochene Wort allein lebendig wirke, sollten allgemein verständliche Vorträge in den zum damaligen Zeitpunkt neuerdings fast überall mit den Museen verbundenen Hörsälen abgehalten und Führungen durch die Sammlungen veranstaltet werden. Vgl. von Stengel/Fleischmann, Wörterbuch des deutschen Staatsund Verwaltungsrechts, 2. Bd, 2. Aufl., 1913, S. 914 f. (Stichwort: Museen, öffentlich).

Diese Aufgabenstellung von Museen, bestimmte Objekte auszuwählen, zu erhalten, zu erforschen und öffentlich zu präsentieren, charakterisiert auch heute den Museumsbegriff. Vgl. Waidacher, Museologie - knapp gefasst, 2005, S. 11,14, 49, 121; Meyers Enzyklopädisches Lexikon, 1976, Bd. 16, S. 632 (Stichwort Museum); dtv-Lexikon 1990, Bd. 12, S. 257 (Stichwort: Museum).

Der International Council of Museums (ICOM) beschreibt die Funktion und die Aufgaben eines Museums damit, dass es sich um eine gemeinnützige, ständige, der Öffentlichkeit zugängliche Einrichtung im Dienst der Gesellschaft und ihrer Entwicklung handelt, die zu Studien-, Bildungs- und Unterhaltungszwecken materielle Zeugnisse von Menschen und ihrer Umwelt beschafft, bewahrt, erforscht, bekannt macht und ausstellt. Dementsprechend beginnt der Katalog der ethischen Richtlinien für Museen des ICOM unter Nr. 1 mit der Rubrik "Museen bewahren, zeigen, vermitteln und fördern das Verständnis für das Natur- und Kulturerbe der Menschheit“. Vgl. die Internetveröffentlichung unter www.icom-deutschland.de.

Dass die Klägerin die so beschriebenen, museumstypischen Leistungen einerseits der Objektbeschaffung und andererseits der musealen Vermittlung und Präsentation der Objekte erfüllt, steht außer Zweifel.

Bereits der Gesellschaftsvertrag stellt in $§ 2$ Abs. 1 und 2 diese kulturelle Aufgabenstellung in den Mittelpunkt, in dem dort ausgeführt ist:

Gegenstand des Unternehmens ist es, die Kunst- und Ausstellungshalle der Bundesrepublik Deutschland zu betreiben und geistige und kulturelle Entwicklungen von nationaler und internationaler Bedeutung sichtbar zu machen, insbesondere durch Ausstellungen, die von der Gesellschaft veranstaltet oder auch im Austausch übernommen werden. Vor allem im Zusammenhang mit Ausstellungen gemäß Abs. 1 kann die Gesellschaft auch Vorträge, Diskussionen, Film- und Musikaufführun- gen sowie andere Präsentationen veranstalten. Die Gesellschaft steht auch als Forum für Gespräche zwischen Persönlichkeiten aus Kunst, Kultur und Geistesleben sowie aus dem Bereich der Politik zur Verfügung.

Zu den Ausstellungen werden noch ein Archiv und eine Bibliothek betrieben, wodurch die wissenschaftliche Durchdringung der Objekte gefördert wird. Das Organigramm der Klägerin sieht neben dem Bereich Projektmanagement (Ausstellungs- und Projektleitung) die Bereiche Unternehmenskommunikation (Publikationen, Lektorat/Textredaktion) und Kunstvermittlung/Bildung vor. Auch dies entspricht den museumstypischen Aufgaben der wissenschaftlichen Begleitung von Ausstellungen einerseits und der Volksbildung andererseits.

Soweit es den Inhalt der von der Klägerin erbrachten Leistungen betrifft - vgl. die Definition des "Museumsbegriffs" in § 4 Nr. 20 Buchst. a) S. 4 UStG, dürften diese zum einen dem Wissenschaftscharakter der Aufgabenerfüllung entsprechen. Dies gilt etwa für die Ausstellung „Auf den Spuren der Irokesen“ in der Zeit vom 22. März bis zum 4. August 2013. Insbesondere lässt sich jedoch der Kunstcharakter der Aufgabenerfüllung feststellen. Insoweit hat bereits das Verwaltungsgericht im erstinstanzlichen Urteil folgende Veranstaltungen aufgeführt:

- „Amedeo Modigliani“ vom 17. April bis 30. August 2009;

- "Gipfeltreffen der Moderne, Das Kunstmuseum Winterthur, Die Großen Sammlungen“ vom 24. April bis 23. August 2009;

- "Markus Lüpertz, Hauptwege und Nebenweg, Eine Retrospektive. Bilder und Skulpturen von 1963 bis 2009" vom 9. Oktober 2009 bis 17. Januar 2010;

- "Neugierig? Die Kunst des 21. Jahrhunderts aus privaten Sammlungen" vom 29. Januar bis 02. Mai 2010;

- „Arp. Beckmann, Munch, Kirchner, Warhol ... Klassiker in Bonn, Die unbekannte Sammlung aus Bielefeld" vom 28. Januar bis 27. März 2011;

- "Max Liebermann, Wegbereiter der Moderne" vom 21. April bis 11. September 2011;

- "Ménage à trois, Warhol, Basquiat, Clemente" vom 10. Februar bis 20. Mai 2012;

- "Sammlung zeitgenössischer Kunst der Bundesrepublik Deutschland, Ankäufe von 2007 bis 2011" vom 18. Januar bis 14. April 2013.

Diese Aufstellung lässt sich um weitere Veranstaltungen erweitern, wie:

- „Echoraum IX „Auflauf der Fassaden“ vom 17. Mai bis 06. Oktober 2013; 
- „Kleopatra, Die ewige Diva“ vom 28. Juni bis 06. Oktober 2013;

- „1914, Die Avantgarde im Kampf“ vom 08. November 2013 bis zum 23. Februar 2014.

Angesichts des Umfangs dieser Ausstellungstätigkeit der Klägerin kommt ihrem Vorbringen, ihr Aufgabenbereich habe sich insoweit gewandelt, als sie sich in größerem Umfang mit der Planung und Durchführung von Ausstellungen anderer Einrichtungen u.ä. beschäftige, keine streitentscheidende Bedeutung zu. Nach wie vor liegt das Kerngeschäft der Klägerin in der Zusammenstellung von Ausstellungsobjekten und deren wissenschaftlicher und volksbildungsorientierter Präsentation.

Der Annahme, dass die Klägerin gleichartige kulturelle Aufgaben wie die Museen in öffentlich-rechtlicher Trägerschaft erfüllt, steht der Umstand nicht entgegen, dass sie über keine eigenen und ständigen Sammlungen verfügt. Zwar bestehen nach Auffassung des Senats durchaus Zweifel, ob mangels einer eigenen Sammlung der Museumsbegriff des $\S 4 \mathrm{Nr} .20$ Buchst. a) S. 4 UStG erfüllt ist. Für die Frage der Gleichartigkeit der kulturellen Aufgabenerfüllung, hat das Fehlen einer eigenen und ständigen Sammlung indes keine Bedeutung.

Die von der Klägerin vorgenommenen Präsentationen von Ausstellungsstücken setzten nämlich voraus, dass eine Sammlung vorhanden ist, sei es durch die Übernahme von bestehenden Sammlungen anderer Einrichtungen, sei es durch die ad hoc zusammengestellte vorübergehende Sammlung von durch verschiedene Einrichtungen zur Verfügung gestellten Objekten, die nach Abschluss der Präsentation wieder zurückgegeben oder aufgelöst wird. Eine selbständige, für die Annahme gleicher kultureller Aufgabenerfüllung entscheidende Bedeutung hat eine eigene und ständige Sammlung nicht. Die Aufgabe eines Museums liegt - wie oben ausgeführt - in der Präsentation und Vermittlung von Ausstellungsstücken für die Wissenschaft und das Laienpublikum. Das Sammeln und Bewahren von Objekten ist für ein Museum nie Selbstzweck, sondern Mittel zum eigentlichen Museumszweck, Ausstellungsobjekte für die Wissenschaft und das Laienpublikum zu präsentieren und zu vermitteln. Das bloße Sammeln und Bewahren von Objekten zeichnet den Privatsammler aus, der keinerlei Museumsaufgaben erfüllt, sondern sich nur des Mittels einer Sammlung bedient, um sein Sammelinteresse zu befriedigen. Weil eine Sammlung, sei es eine eigene oder eine mit fremden Objekten, sei es eine ständige oder eine nur zeitlich beschränkte, lediglich das Mittel zur kulturellen Aufgabenerfüllung von Museen ist, handelt es sich um ein Einrichtungs- und kein Aufgabenmerkmal, dessen notwendige Eigenschaften im Rahmen des Museumsbegriff festzulegen die Finanzverwaltung und Finanzgerichtsbarkeit berufen sind.

Liegen somit im Fall der Klägerin die tatbestandlichen Voraussetzungen für die Erteilung der Bescheinigung nach § 4 Nr. 20 Buchst. a) S. 2 UStG - weiterhin - vor mit der Folge, dass kein Anspruch der Klägerin auf deren Aufhebung besteht, ist die Berufung mit der sich aus § 154 Abs. 2 VwGO ergebenden Kostenfolge zurückzuweisen.

Die Revision ist nicht zuzulassen, da die Voraussetzungen des § 132 Abs. 2 VwGO nicht vorliegen. (Entscheidung von der Redaktion bearbeitet.)

Anzeige

\section{KUNST AUKTIONSKATALOGE} ZU VERKAUFEN

\author{
1890 bis 1950 \\ $500+$ Volumen \\ $\mathrm{DE}-\mathrm{CH}-\mathrm{FR}-\mathrm{GB}$
}
Aus bedeutender $\mathrm{CH}-\mathrm{Galerie}$. Viele mit Ergebnislisten. Kontakt: auktionskataloge@gmail.com

